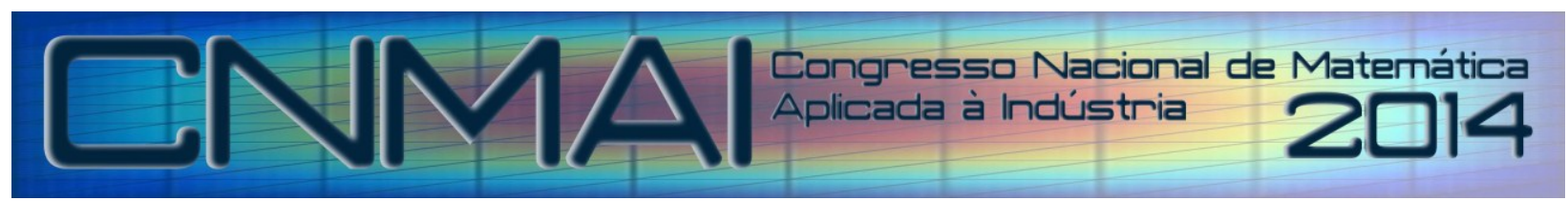

18 a 21 de novembro de 2014, Caldas Novas - Goiás

\title{
MÉTODO DA MÁXIMA ENTROPIA APLICADO AO DESENVOLVIMENTO DE MODELO PROBABILÍSTICO DE PARÂMETROS DE CIRCUITOS ELÉTRICOS SHUNT
}

\author{
Bruno Gabriel Gustavo Leonardo Zambolini Vicente, brunozv@mec.ufu.br \\ Victor Augusto da Costa e Silva, victor_augusto_cn@hotmail.com \\ Antônio Marcos Gonçalves de Lima, amglima@mecanica.ufu.br
}

\begin{abstract}
${ }^{1,2,3}$ Universidade Federal de Uberlândia - Faculdade de Engenharia Mecânica, Campus Santa Mônica, Uberlândia-MG
Resumo: No presente trabalho apresenta-se a construção do modelo probabilístico dos parâmetros de circuitos elétricos shunt aplicado a estruturas compostas contendo materiais piezelétricos no contexto de controle passivo de vibrações. Para isso, o Método da Máxima Entropia foi usado como ferramenta matemática para obtenção das funções densidade de probabilidade que modelam o comportamento probabilístico de cada parâmetro em análise. Neste sentido, ênfase foi dada à abordagem paramétrica, uma vez que as incertezas serão introduzidas diretamente nos parâmetros do modelo, sendo também realizada a análise da influência de diferentes níveis de incertezas em cada um dos parâmetros dos circuitos shunt. Através dos resultados das análises, foram estabelecidos os limites de incertezas, o número mínimo de amostras e as prováveis distribuições de probabilidades para cada variável de projeto a serem utilizadas no processo de otimização robusta dos circuitos elétricos shunt, realizado a posteriori.
\end{abstract}

Palavras-chave: modelagem probabilística, Método da Máxima Entropia, circuitos elétricos shunt, materiais piezelétricos, estruturas compostas.

\section{INTRODUÇÃO}

No contexto de estruturas inteligentes e seguindo os trabalhos de Soize (2010) e Ritto et al. (2008), o presente artigo apresenta avaliação da influência de incertezas nos parâmetros de resistência e indutância do circuito shunt e espessura do material piezelétrico de uma estrutura composta contendo material piezelétrico associado a circuito elétrico shunt ressonante. Através do Método da Máxima Entropia, determinou-se a distribuição apropriada do comportamento estocástico das incertezas e fundamentou-se o nível de dispersão máximo e de número de tiragens para convergência do modelo. Essas informações são de fundamental importância para o desenvolvimento do projeto ótimorobusto de sistema para mitigação de vibrações, tais como o trabalho realizado por Santos (2012).

O processo de avaliação dos parâmetros baseia-se na análise da função de resposta em frequência (FRF) da estrutura contendo elementos piezelétricos combinados com circuitos shunt, dada por:

$$
H(\omega)=\left[K_{u u}-K_{u \varphi}\left(K_{\varphi \varphi}-\frac{1}{j \omega} Z^{-1}(\omega)\right)^{-1} K_{\varphi u}-\omega^{2} M\right]^{-1}
$$

Onde $K_{u u}$ é a rigidez puramente mecânica, $K_{u \varphi}$ e $K_{\varphi u}$ são as rigidezes eletromecânicas, $K_{\varphi \varphi}$ é a rigidez puramente elétrica e $M$ é a massa do sistema. A Eq. (1) pode ser utilizada para diferentes tipos de circuitos shunt mediante a introdução das expressões correspondentes de suas impedâncias elétricas, indicadas por $Z(\omega)$.

\section{MODELO NOMINAL}

Estabeleceu-se como modelo nominal, denominado por $\bar{H}(\omega)$, uma viga em balanço de quatro camadas de material composto, esquematizada na Fig. (1), contendo um elemento PZT do tipo PZT G1195, na posição indicada, combinada a dois circuitos elétricos shunt: resistivo e ressonante. 


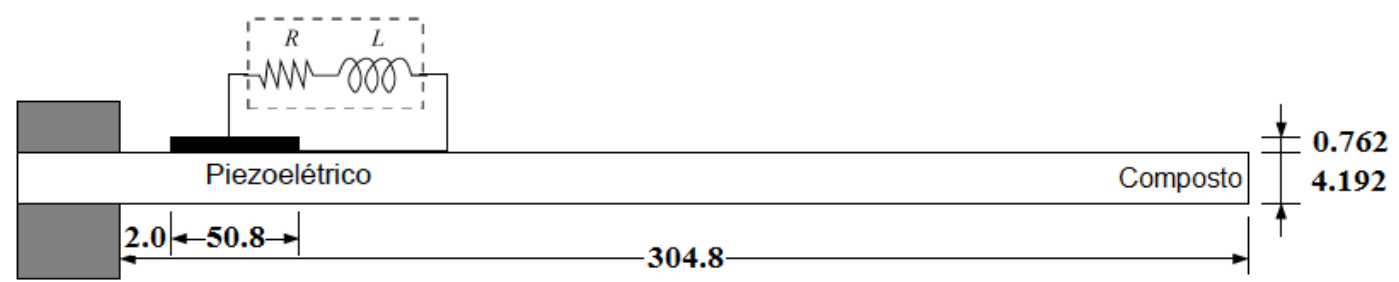

Figura 1 - Placa composta contendo circuito elétrico shunt ressonante.

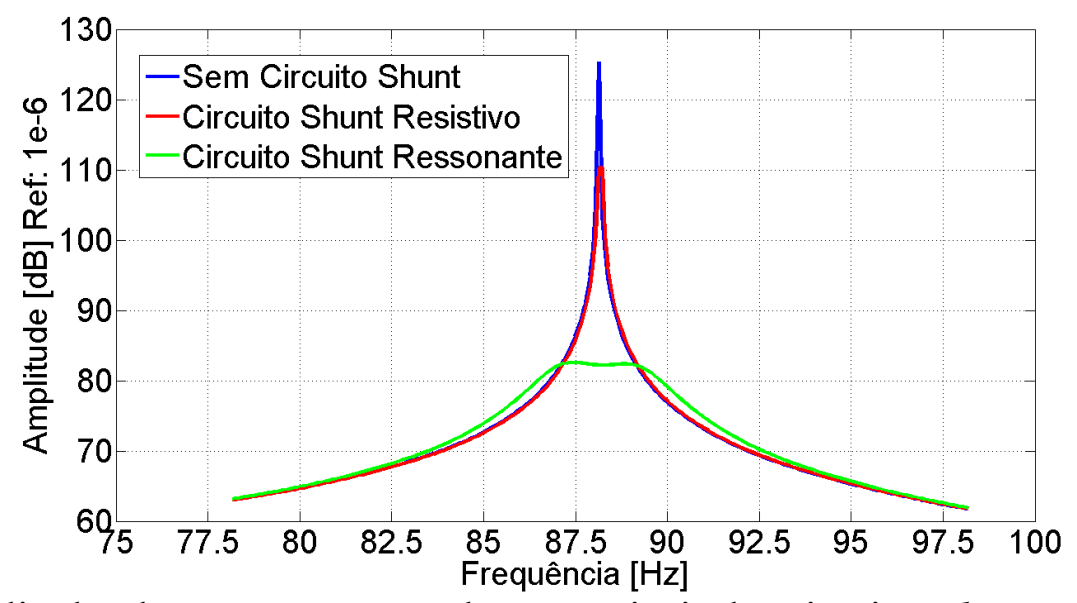

Figura 2 -Amplitudes das FRFs para os valores nominais dos circuitos shunt analisados.

Inicialmente, a espessura do PZT $\left(h_{P Z T}\right)$ foi considerada igual a $0,762 \mathrm{~mm}$ e a resistência do circuito shunt resistivo igual a $3,23 \times 10^{5} \Omega$. Para o circuito shunt ressonante assumiu-se $R=1,61 \times 10^{4} \Omega$ e $L=6,29 \times 10^{2} \mathrm{H}$. Sabendo que o circuito shunt resistivo impõe $Z(\omega)=R$ e o shunt ressonante determina $Z(\omega)=R+j \omega L$ na Eq. (1), a Fig. (2) apresenta as amplitudes das FRFs obtidas para o primeiro modo de vibração, onde nota-se a maior redução nível de vibração do circuito elétrico shunt ressonante, sendo essa configuração a escolhida para o processo de modelagem estocástica com vistas na otimização robusta dos parâmetros.

\section{QUANTIFICAÇÃO DE INCERTEZAS}

O processo de construção do modelo probabilístico para cada parâmetro de projeto baseou-se no Método da Máxima Entropia (MME), que se apoia no fato de que ao maximizar a entropia do sistema, maximizam-se também as incertezas contidas no mesmo, o que resulta no mínimo de informações inferidas (Shannon, 1948). Portanto, partindo-se de distribuições que atendem as restrições (por exemplo, os momentos estatísticos) de uma dada variável de projeto, o MME tem determina a distribuição que apresenta o máximo de incerteza, ou seja, a máxima entropia.

Segundo Shannon (1948), dada uma função densidade de probabilidade $p_{x}(x)$, sua entropia é dada pela Eq. (2):

$$
S\left(p_{x}(x)\right)=-\int p_{x}(x) \ln \left(p_{x}(x)\right) d x
$$

Do ponto de vista da matemática estatística, frequentemente conhece-se alguma informação sobre $x$, como sua média e/ou sua variância, sendo essas informações descritas como momentos estatísticos Usando essas informações e aplicando multiplicadores de Lagrange, maximiza-se o seguinte funcional $p_{x}(x)$ :

$$
\delta \bar{S}=\int\left[-\ln \left(p_{x}(x)\right)-1+\sum_{i=0}^{n} \lambda_{i} x^{i}\right] \delta p_{x}(x) d x=0
$$

A Eq. (3) fornece a expressão para a provável função densidade de probabilidade (FDP) da variável aleatória $x$ :

$$
p_{x}(x)=\exp \left[-1+\sum_{i=0}^{n} \lambda_{i} x^{i}\right]
$$


Onde os multiplicadores de Lagrange, $\lambda_{i}$, podem ser obtidos através das $n+1$ equações de restrições impostas pelos momentos estatísticos conhecidos, para a variável aleatória $x$.

Seguindo o trabalho de Soize (2010), observa-se que os parâmetros em análise, a saber, $R, L$ e $h_{P Z T}$ compartilham propriedades físicas elementares: são essencialmente positivos, limitados e não-nulos. Representando de forma generalizada esses parâmetros através da variável aleatória $X$, considera-se as seguintes restrições: (i) admite valores no intervalo $X \in] 0,+\infty[$; (ii) é conhecida a média, $E\{X\}=\bar{X}$; (iii) a dispersão é finita, $E\{\ln (X)\}=c$, onde $|c|<+\infty$ (Soize, 2010). Resolvendo a Eq. (4) e impondo-se essas restrições, obtém-se a seguinte função Gamma:

$$
p_{X}(x)=1_{] 0,+\infty}(x) \frac{1}{\bar{X}}\left(\frac{1}{\delta_{X}^{2}}\right)^{\frac{1}{\delta_{X}^{2}}} \frac{1}{\Gamma\left(\frac{1}{\delta_{X}^{2}}\right)}\left(\frac{x}{\bar{X}}\right)^{\frac{1}{\delta_{X}^{2}}-1} \exp \left[-\frac{x}{\delta_{X}^{2} \bar{X}}\right] \quad, \quad \Gamma(z)=\int_{0}^{+\infty} t^{z-1} \exp (-t) d t
$$

Onde $\delta_{X}=\sigma_{X} / \bar{X}$ é a dispersão relativa de $X$, limitada a $0<\delta_{X}<1 / \sqrt{3}$ (Ritto $e t$ al, 2008), ou seja, de 0 a $57,7 \%$.

A fim de se avaliar o modelo probabilístico obtido na Eq. (6) para os parâmetros em análise, empregou-se a função $\operatorname{GAMRND}(\alpha, \beta)$ do $\operatorname{MATLAB}^{\circledR}$, sendo $\alpha=1 / \delta_{X}^{2}$ e $\beta=\bar{X} \delta_{X}^{2}$, de acordo com o trabalho de (Ritto et al, 2008) e investigou-se o número mínimo de tiragens aleatórias para a convergência usando a seguinte função de convergência:

$$
\operatorname{conv}\left(n_{s}\right)=\frac{1}{n_{s}} \sum_{j=1}^{n_{s}}\left\|H_{j}(\omega, \theta)-\bar{H}(\omega)\right\|^{2}
$$

Sendo $n_{s}$ o número de realizações, $H_{j}(\omega, \theta)$ a jésima FRF para uma dada realização aleatória $\theta$.

\subsection{Modelagem estocástica de incertezas nos parâmetros e avaliação de convergência}

Conforme Sedra (2009), os resistores e indutores disponíveis comercialmente apresentam, no pior caso, 20\% de tolerância em relação ao seu valor nominal. Assim, escolheu-se a máxima dispersão relativa desses parâmetros como $\delta_{R, L}^{\max }=0,3$, ou seja, abaixo do limite teórico de 57,7\%. Segundo Selten (2005), as incertezas na espessura dos materiais piezelétricos dependem da qualidade no processo de fabricação do mesmo. Partindo-se de catálogos disponíveis dos fabricantes PI - Piezotechnology ${ }^{\circledR}$ e Midé Technology Corp. ${ }^{\circledR}$, a tolerância na espessura pode atingir $5 \%$, escolheu-se o valor limite da dispersão relativa em $10 \%$, ou seja, $\delta_{h_{P Z T}}^{\max }=0,1$, já que, segundo Andreaus (2007), valores de incertezas superiores acarretariam em modificação estrutural, descaracterizando o problema original.

Para o modelo nominal foram calculados os parâmetros $\alpha$ e $\beta$ a serem empregados na FDP Gamma para a avaliação da convergência (Eq. (6)) e, para cada nível de dispersão realizou-se 5000 tiragens aleatórias, usando o método do Hyper-Cubo-Latino (HCL), variante do Método de Monte Carlo, com garantia de convergência mais rápida.

A Figura (3) apresenta as FDPs para cada dispersão, em cada parâmetro. Nota-se que para valores acima de $10 \%$ as funções Gamma apresentam deslocamento do pico e achatamento dos valores de probabilidade.
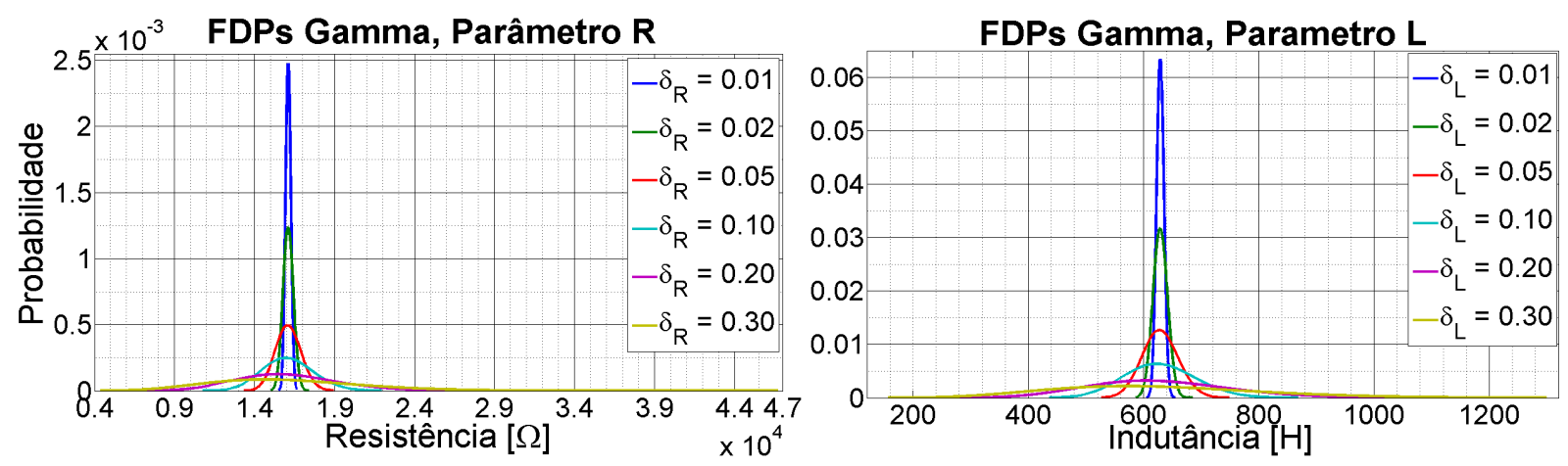


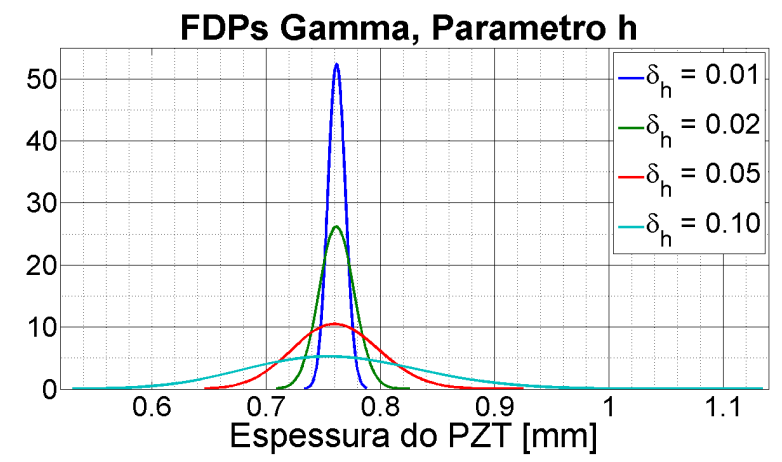

(c)

Figura 3 - Distribuições Gamma para diferentes valores de dispersão para cada parâmetro.

As Figuras (4) exibem os envelopes das FRFs para algumas das dispersões em cada um dos parâmetros. Deve-se salientar que para avaliação das incertezas fez-se variar um parâmetro por vez, mantendo os demais constantes. Observando as Figs. (4.a) a (4.c), FRFs para incerteza em $R$, nota-se que quanto maior o nível de dispersão $\delta_{R}$, maior é a dispersão ao redor da resposta média do sistema. Nas Figuras (5.a) a (5.c) nota-se que um aumento no nível de dispersão $\delta_{L}$ do parâmetro $L$ acarreta um aumento do intervalo de confiança e, em relação ao parâmetro $R$, exerce maior grau de influência na atenuação de vibrações. Nas Figs. (6.a) a (6.c) observa-se que a influência das incertezas no parâmetro espessura do elemento piezelétrico, $h_{P Z T}$, é similar à influência das incertezas no parâmetro de indutância.

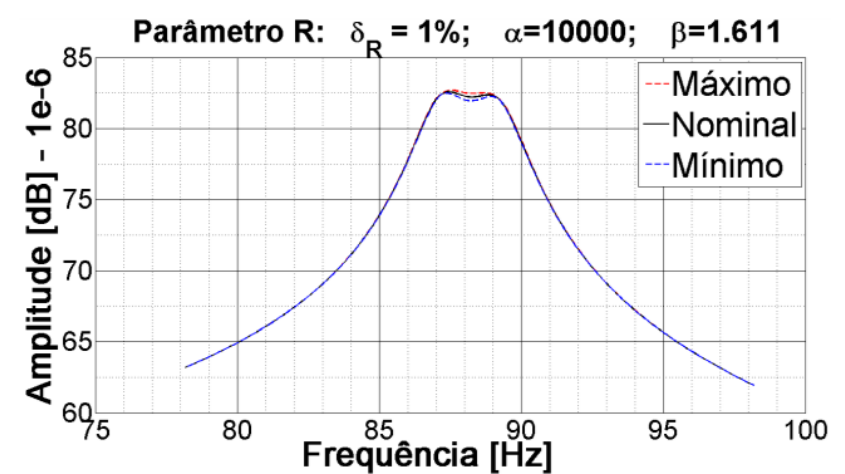

(a)

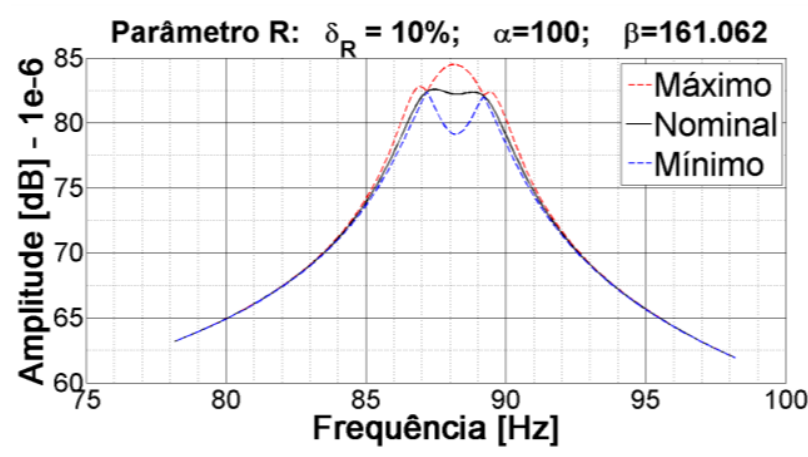

(b)

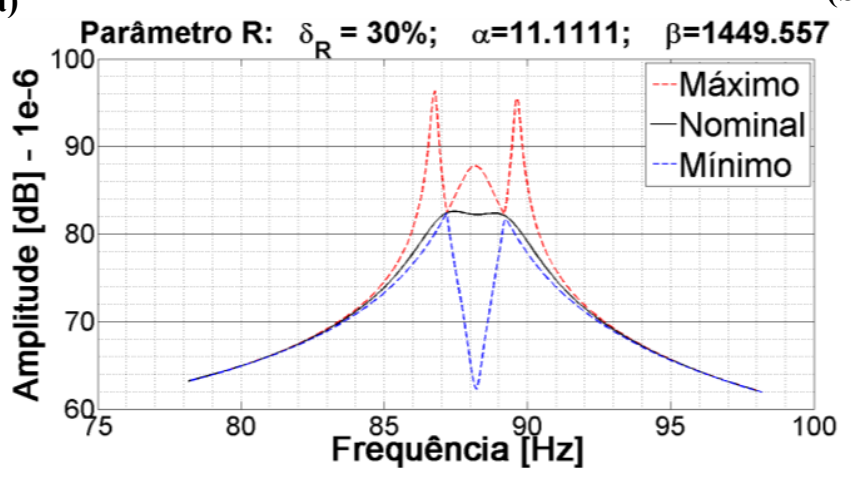

(c)

Figura 4 - Envelopes das FRFs para incertezas nos parâmetros $R$.

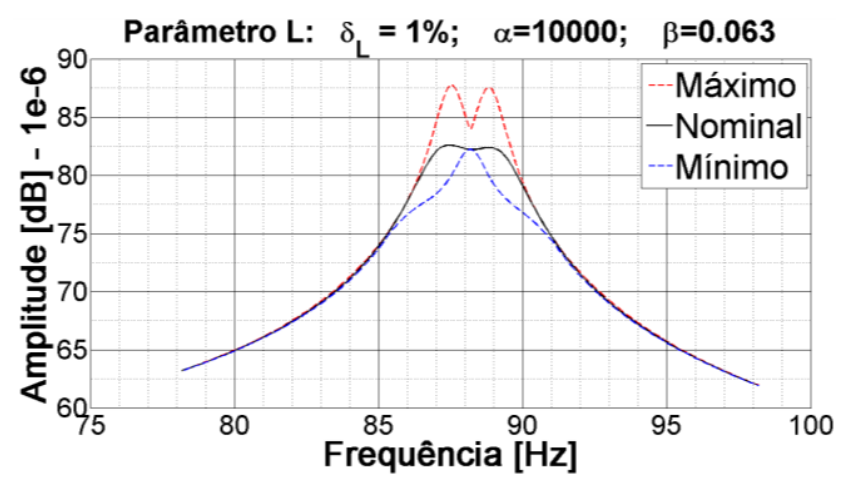

(a)

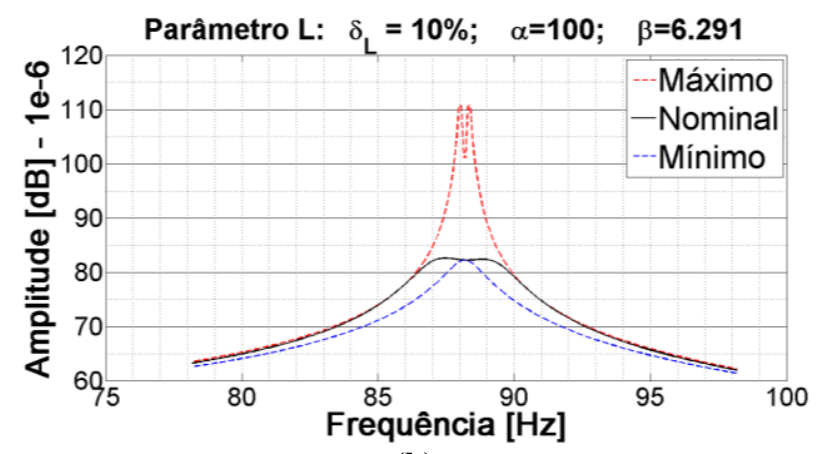

(b) 


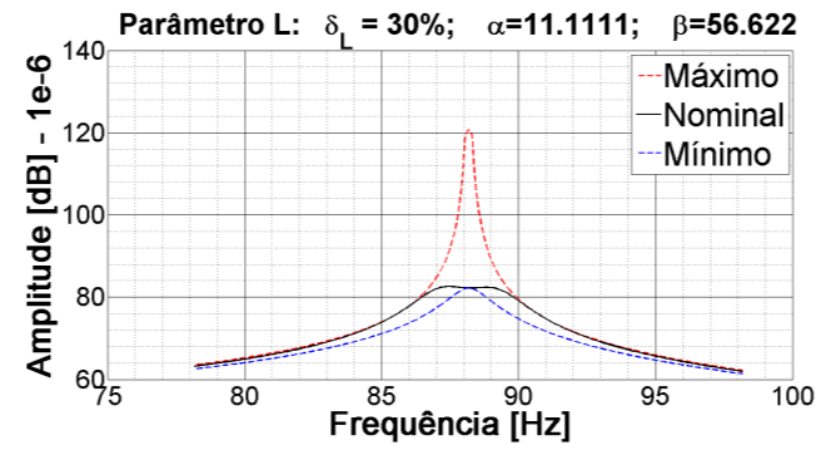

(c)

Figura 5 - Envelopes das FRFs para incertezas no parâmetro $L$.

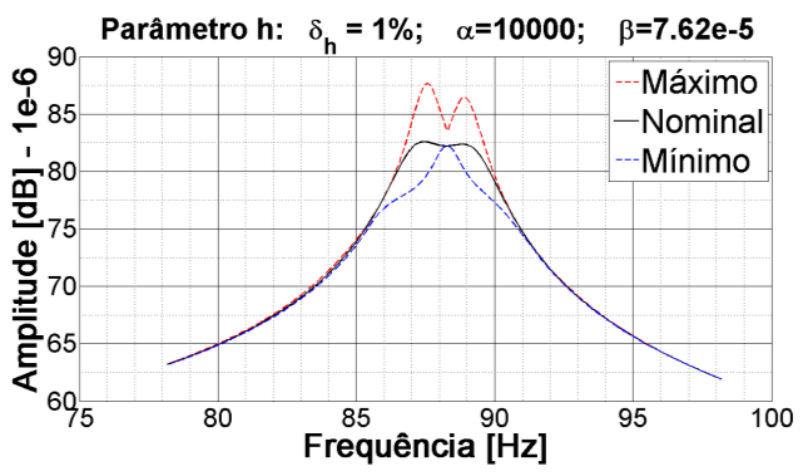

(a)

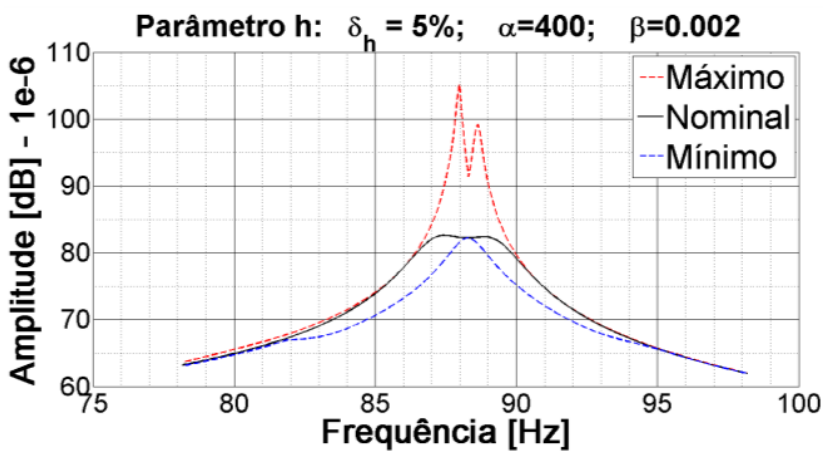

(b)

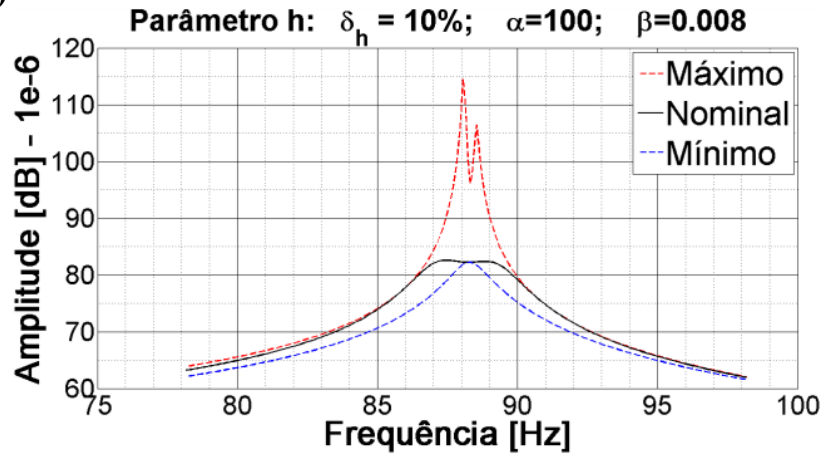

(c)

Figura 6 - Envelopes das FRFs para incertezas no parâmetro $h_{P Z T}$.

A Figuras 7, 8 e 9 apresentam a evolução do erro quadrático médio versus número de tiragens aleatórias. Para o parâmetro $R$, Figuras (7), nota-se que foi necessário no pior caso (dispersão de 30\%) 1500 realizações para convergência. No caso do parâmetro $L$, Figuras (8), observa-se que em alguns casos são necessárias 2500 realizações para convergência. Já o parâmetro $h_{P Z T}$, Figuras (9), apresentou a pior situação de convergência para 5\% de dispersão, sendo necessário pelo menos 3000 realizações.

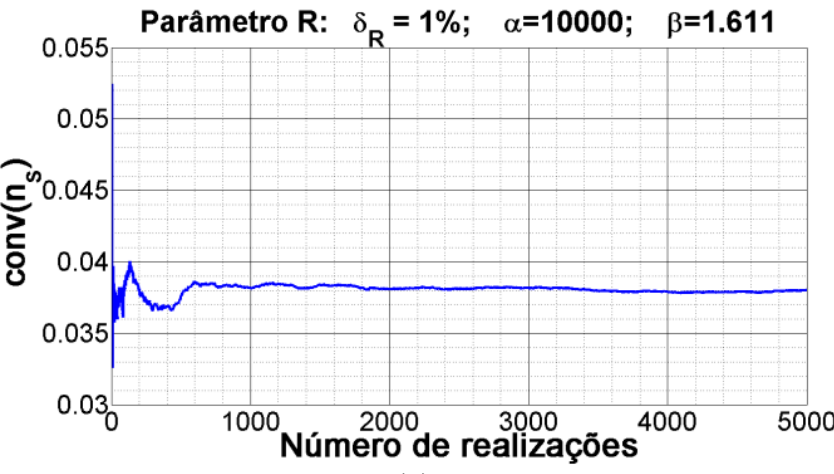

(a)

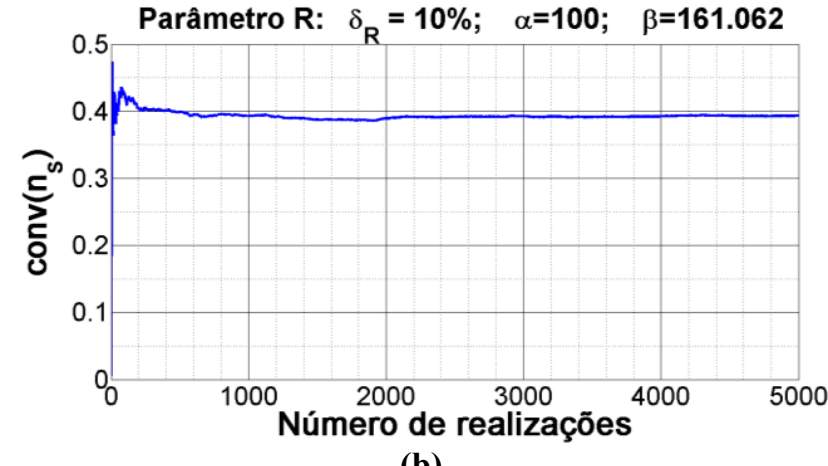

(b) 


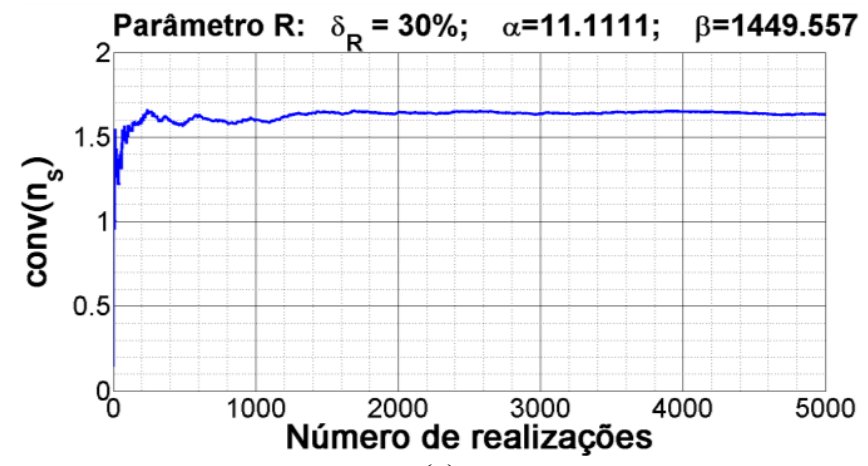

(c)

Figura 7 - Convergência do erro quadrático médio no parâmetro $R$.

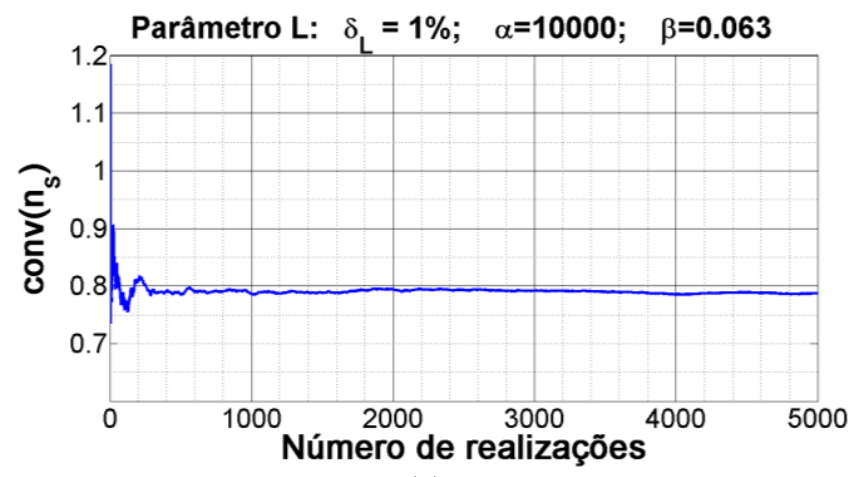

(a)

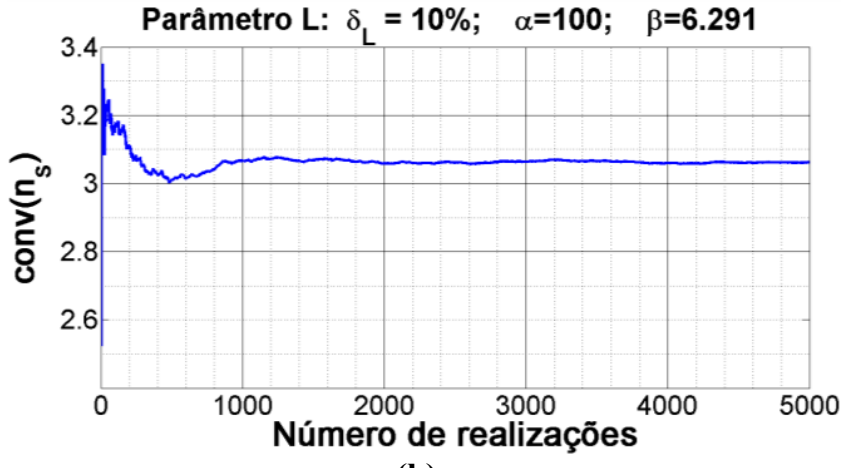

(b)

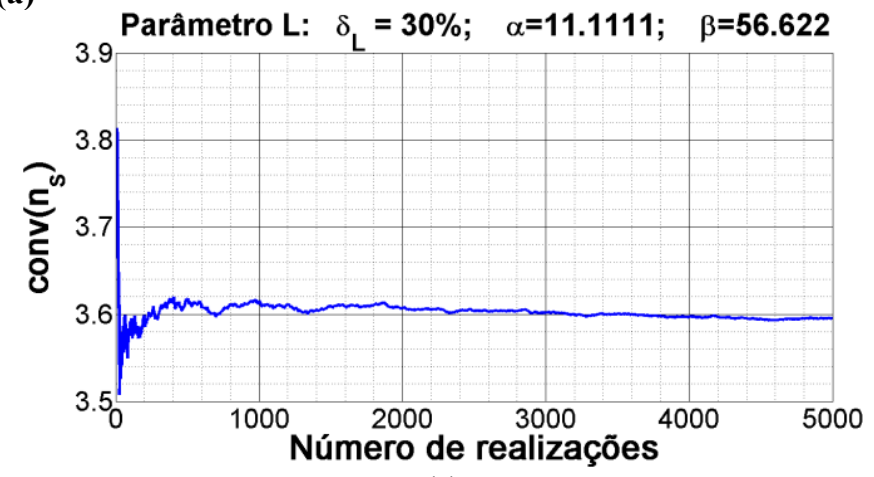

(c)

Figura 8 - Convergência do erro quadrático médio no parâmetro $L$.

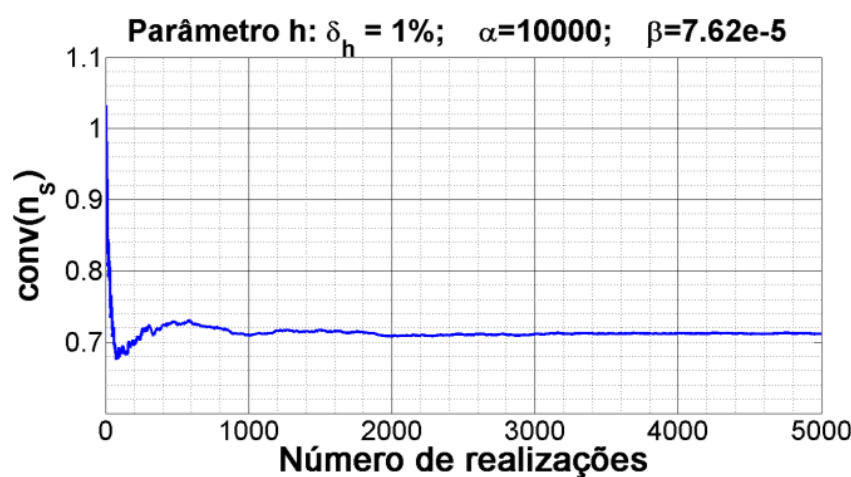

(a)

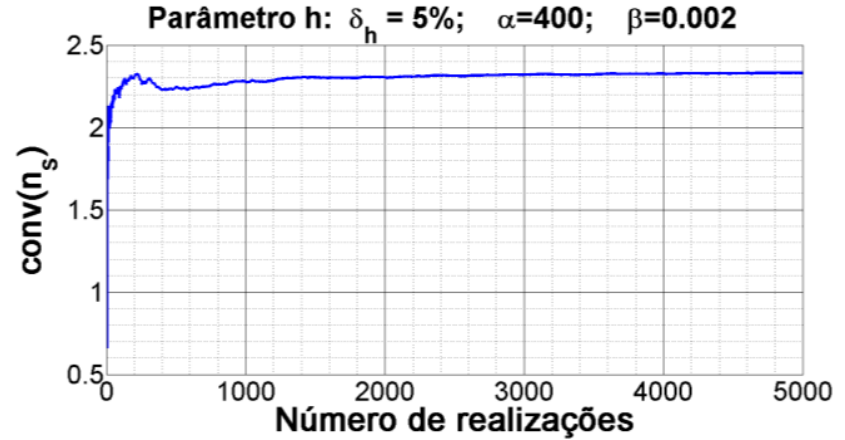

(b) 


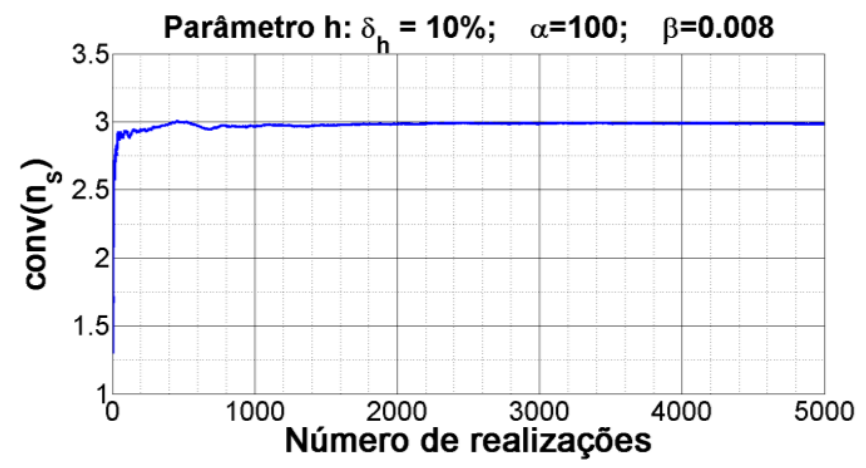

(c)

Figura 9 - Convergência do erro quadrático médio no parâmetro $h_{P Z T}$.

A partir das análises realizadas, a Tab. (1) apresenta os limites de dispersão e do número mínimo de tiragens aleatórias consolidados, a serem utilizados posteriormente no processo de otimização robusta:

Tabela 1 - Dispersão, $\delta$, e número de tiragens, $n$.

\begin{tabular}{|c|c|c|c|}
\cline { 2 - 4 } \multicolumn{1}{c|}{} & $R$ & $L$ & $h_{P Z T}$ \\
\hline$\delta$ & 0,30 & 0,30 & 0,10 \\
\hline$n$ & 1500 & 2500 & 3000 \\
\hline
\end{tabular}

\title{
4. REFERÊNCIAS
}

Andreaus, U.; Porfiri, M., 2006, "Effect of Electrical Uncertainties on Resonant Piezoelectric Shunting". In Journal of Intelligent Material Systems and Structures, May 2007 18: 477-485, first published on October 30.

Ritto, T. G., Sampaio, R. Cataldo, E. 2008, "Timoshenko Beam with Uncertainty on the Boundary Conditions", In Journal of Brazilian Society of Mechanical Science e Engineering, Oct-Dec, Vol. XXX, No. 4, pp. 295-303.

Santos, H. F. L., 2012, Controle ativo-passivo de vibrações estruturais usando materiais piezelétricos: Otimização e quantificação de incertezas, Tese de Doutorado - EESC/USP, São Carlos, SP.

Shannon C. E., A mathematical theory of communication, 1948, Bell System Tech. J., 27, 379-423 and 623-659.

Sedra, A. S.; Smith, K. C. Microeletrônica, 2009, 6ª Edição / Editora: Oxford, ISBN13: 9780195323030 ,

Selten, M., Schneider, G.A., Knoblauch, V. and McMeeking, R. M., 2005, "On the evolution of the linear material properties of PZT during loading history: an experimental study", In Journal of Solids and Structures, 1-14.

Soize, C., 2010, "Stochastic Models in Computational Mechanics", Seminar on Uncertainties and Stochastic Modeling, PUC-Rio, August, 2-6.

\section{RESPONSABILIDADE PELAS INFORMAÇÕES}

Os autores são os únicos responsáveis pelas informações incluídas neste trabalho.

\section{MAXIMUM ENTROPY PRINCIPLE APPLIED TO DEVELOPMENT OF SHUNT CIRCUITS PROBABILISTIC MODEL PARAMETERS}

\author{
Bruno Gabriel Gustavo Leonardo Zambolini Vicente, brunozv@mec.ufu.br \\ Victor Augusto da Costa e Silva, victor_augusto_cn@hotmail.com \\ Antônio Marcos Gonçalves de Lima, amglima@mecanica.ufu.br
}

\footnotetext{
${ }^{1,2,3}$ Federal University of Uberlândia - Mechanical Engineering Faculty, Campus Santa Mônica, Uberlândia-MG
}

\begin{abstract}
In this paper is presented the construction of the probabilistic model of the electrical circuit parameters shunt applied to composite structures containing piezoelectric materials in the context of passive vibration control. For this, the Maximum Entropy Principle was used as a mathematical tool for obtaining the probability density functions that model the probabilistic behavior of each parameter in analysis. In this regard, emphasis was placed on parametric approach, since the uncertainties are introduced directly in the model parameters, which is also performed the analysis of the influence of different levels of uncertainty in each parameter of the shunt circuits. Through the analysis results, were established limits of uncertainty, the minimum number of samples and the likely probability distributions for each design variable to be used in robust optimization of shunt circuits process performed retrospectively.

Keywords: probabilistic modeling, maximum entropy principle, shunt circuits, piezelectric materials, composites.
\end{abstract}

